\title{
A Hybrid Communication Platform for Multi- Microgrid Energy Management System Optimization
}

\author{
Mojtaba Moghimi ${ }^{1,3}$, Pouya Jamborsalamati², Jahangir Hossain², Sascha Stegen ${ }^{3}$, Junwei Lu $^{1,3}$ \\ ${ }^{1}$ Queensland Micro and Nanotechnology Centre, Griffith University, Brisbane, Australia \\ ${ }^{2}$ School of Engineering, Macquarie University, Sydney, Australia \\ ${ }^{3}$ School of Engineering, Griffith University, Brisbane, Australia \\ m.moghimi@griffith.edu.au
}

\begin{abstract}
This paper proposes the communication platform for Multi-Microgrid (MMG) Energy Management System (EMS) using combination of communication protocols in a hierarchical architecture. There is an Internet of Things (IoT) gateway designed in the proposed platform, which aims to connect multiple Microgrids to each other. Through the designed communication platform, bi-directional data exchange among the MGs for the optimal operation of the MGs could be achieved. Due to the high number of devices required to communicate in MMG optimization problems, a cloud-based server, which enables extensive data sharing and analysis of the collected data, is employed in this work. Modbus protocol is used for the local communication level, i.e. communications between the devices within an individual MG and the MG Central Controller (MGCC). Message Queue Telemetry Transport (MQTT) protocol is adopted for communications between MGCCs and cloud. Furthermore, HTTP requests are the main communication method for interactions with the cloud channels. A virtual wide Area Network emulator (WANem machine) is adopted to emulate network latency in the system. In case of high latency in the network, MGCC takes action on delivering the optimization results for its Microgrid. The efficiency of the implemented platform for the EMS performance of the MMG is shown by comparing the total cost related to the MMG operation in centralized and distributed modes.
\end{abstract}

Keywords- Multi-MicroGrid, Modbus, MQTT, IoT, Cloudbased data sharing

\section{INTRODUCTION}

Microgrids (MGs) are non-separable blocks of future power grids with the distributed energy resources [1]. Advanced technology brings new potential operation and management of MGs with high number of smart devices connected to a network [2]. Hence, a reliable communication platform, which secures automated data acquisition and supervisory of the data in large scale is vital. For a Multi-Microgrid (MMG) system, this platform is required to realize two layers of communications. The first layer is the communication inside every single MG between the devices and the local MG controller. The second layer of communications aims to coordinate the operation of MGs with respect to each other in the MMG [1]. Therefore, a hybrid comprehensive communication platform is required.

MGs control systems can be designed in centralized or distributed fashions [1]. In centralized mode, all the information from each Intelligent Electronic Device (IED) in each MG is collected in the cloud and the Energy Management System (EMS) with optimization algorithms runs on top of the cloud considering aggregated information [3]. The decision-making procedure in the centralized mode is more complex, consumes more time, and heavily relies on communication reliability [3]. In contradiction, multiple local EMS run in the distributed mode independently. In distributed mode, local controllers collect data from all IEDs and meters in the MG as well as data from other MGs and run the optimization algorithm for that specific MG regardless of the operation conditions of the cloud. The distributed Microgrid EMS decision making comes at a lower cost with less time consumption but gives weaker solutions with lower reliability compared to the centralized fashion [4].

Among the aforementioned control methods, this paper focuses on the design and implementation of a communication platform, which enables evaluation of both distributed and centralized optimization of the MGs. The proposed communication platform facilitates the centralized EMS with MMG environment to coordinate operation of the MGs with respect to each other by minimizing the cost of operation for the MMG. In the proposed hierarchical structure, the centralized optimization runs in the cloud receiving information from multiple local controllers in MMG. If there is communication failure in the system and one of the communication links to the cloud is interrupted or delayed, the local MG central controllers (MGCCs) would react to run local optimizations for individual MGs shifting to the distributed communication mode. Hence, in the proposed architecture, the system comes with results under uncertain conditions even high latency in the network or unreliable communication links.

There are multifarious industrial communications protocols available for control and protection of energy grids. Protocols prescribed by the IEC 61850 standard series are used in works developed in [5-7] for control and protection applications in power transmission and power distribution levels. In addition, DNP3 protocol is adopted by [8] for SCADA applications.

The communication platform designed in this paper utilizes Modbus TCP/IP for communications in the distributed mode when there is no communication to the cloud. Modbus TCP/IP connects IEDs to the MGCC of each MG as well as connecting the MGs to each other as a backup in case of cloud failure or long delay. Message Queue Telemetry Transport (MQTT) protocol, which is the state of the art Internet of Things (IoT) protocol [9], is employed in this paper for communications in centralized fashion with cloud interactions. MQTT optimizes the network power consumption by faster operation of the messages [9]. MQTT is a publisher-subscriber protocol where messages 
are published to customizable topics [9]. MQTT topics include metadata and indicate source of the messages. Therefore, they are proposed in this work according to the MMG scheme. An online cloud server is adopted in this work, which is compatible with MQTT protocol and has interface to MATLAB for postprocess of the collected data from each MG. Hypertext Transfer Protocol (HTTP), which is an application-level protocol over TCP/IP, is used for interactions between each local MGCC and the cloud. HTTP requests (POST and GET) are the core parts of the implementation of the MGCCs.

In order to emulate real characteristics of a network with packet loss and packet delay, a virtual Wide Area Network emulator (WANem machine) is setup in the proposed platform. WANem machine is capable of altering the packet flow path in order to add a customizable delay manually in both wired and wireless environment [8]. Briefly, the hierarchical hybrid platform in this paper utilizes Modbus, MQTT, and HTTP protocols along with an online cloud server and a virtual WANem machine implemented in a co-simulation platform by MATLAB and Python. The proposed communication platform is capable of working in central and distributed modes if any failure happens in the communication hierarchy. In this paper, the impact of the hybrid communication system in presence of communication delay on the EMS of MMG is investigated. In other words, the hierarchical communication system is employed to support the EMS to minimize the operation cost og MMG.

The rest of this paper is structured as follows. Section II presents the MMG platform for distributed and centralized modes. In section III, the results of the MMG in both distributed and centralized fashions along with their impact on the operational costs are indicated. The paper concludes with section IV as the conclusion.

\section{MULTI-MICROGRID PLATFORM DESIGN}

The hybrid hierarchical communication architecture is designed in such a way that it can cover both distributed and centralized operation of the MGs. Centralized fashion is the default operational mode of the MMG in the designed architecture. In case of communication failure and high delay in the communication to the cloud, the distributed mode is activated. There are two main messaging layers. The first layer is between MGCC and the local IEDs. The second layer represents the messaging of the MGs to the cloud via the IoT gateway. These layers are described in details as follows:

\section{A. Distributed mode}

A typical experimental architecture related to a university campus Microgrid is exhibited in Fig. 1. In distributed mode, the decisions are made in the MGCC and sent to the cloud to share with other MGs. Furthermore, the MGs are connected via Modbus TCP/IP to each other that in case of disconnection from the cloud, they still can share data. All IEDs inside the MG use master/slave Modbus protocol, which has a byte-oriented structure [1], to communicate with the MGCC. Backup internal communication protocol between the installed IEDs and the MGCC is HTTP TCP/IP as indicated in Fig. 1. Modbus
Messages are transmitted over Ethernet with the default port of 502. If distributed mode is activated, the information is exchanged between the IEDs within the MGCC required for the energy management algorithm, which runs in the MGCC for that particular MG, and the results go all the way back from MGCC to the IEDs to be actuated. This is achieved by employing Modbus communication protocol in the distributed communication mode.

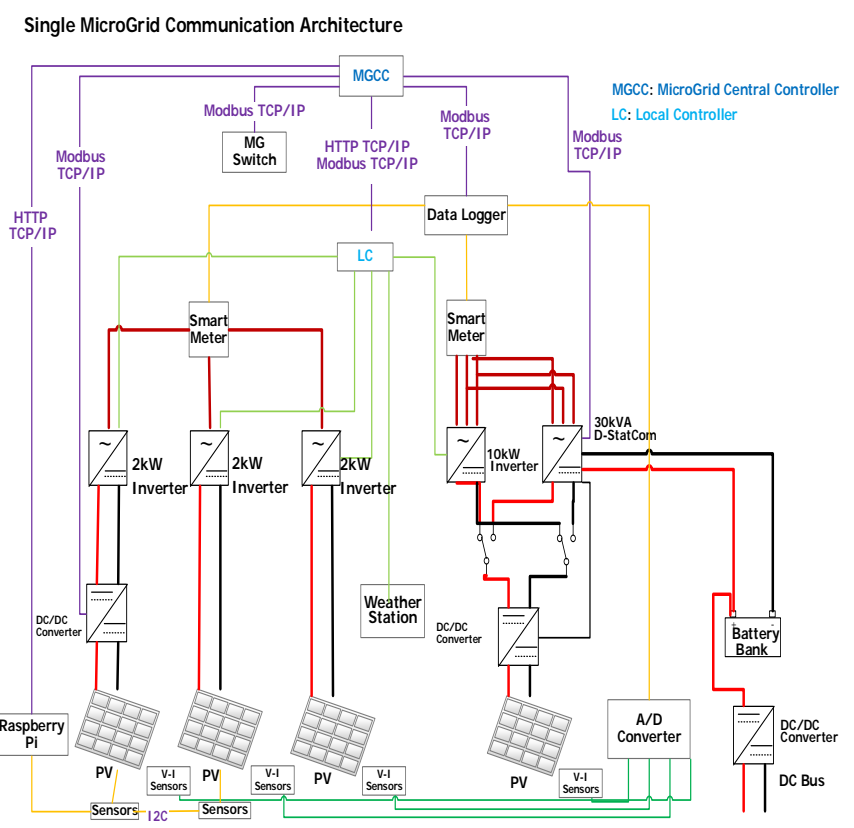

Fig. 1. Overview of MG communication architecture [1]

Fig. 1 shows that all of the inverters connected to the PV panels are connected through a local controller to the MGCC. Data loggers connected to smart meters collect measured data and provide an interface to the MGCC. The purple lines show the flow of communications through Modbus TCP/IP and the red and black lines show the power flow. Other colors represent physical connections among the IEDs inside the MG. As a backup system, sensors are connected to a Raspberry Pi with an interface to the MGCC. In addition, the local controller can provide the MGCC with HTTP TCP/IP connections.

\section{1) Modbus Communication Protocol}

Modbus Application Protocol (MAP), Transmission Control Protocol (TCP), Internet Protocol (IP), Datalink, and Physical layer are five layers in Modbus TCP/IP industrial communication protocol [10]. In server/client Modbus protocol, the destination is defined by IP address and is attached to the message payload. Application Data Unit (ADU) is made of an Application Header, Function Code, and Payload [10]. Four datatypes in Modbus are: 1) single-bit read-only Discrete Input, 2) single-bit coils, 3) 16-bit read-only Input Registers, and 4) 16-bit read-write Holing Registers.

\section{B. Centralized mode}

As described in the beginning of this chapter, the default operational mode of the MGs in this paper is the centralized mode to find the optimum operational condition in coordination 
with other MGs. In centralized fashion, all the IEDs in each MG communicate with the MGCC similar to the distributed mode but the MGCCs communicate with the fellow units in other MGs via a cloud-based IoT gateway. The energy optimization algorithm runs on top of the cloud considering all the collected data in cloud channels in aggregated level. The proposed architecture for centralized MMG energy optimization is shown in Fig. 2. MQTT protocol, which is the favored choice of IoT designers due to its easy-to-use features, is utilized in the centralized fashion to realize messaging among the MGCCs through the cloud.

\section{1) MQTT Protocol}

MQTT communications are realized over TCP/IP with the standard port 1883, which is set to listen to all of the incoming MQTT messages. There are three key players in MQTT messaging:

\section{a) MQTT publisher \\ b) MQTT subscriber \\ c) MQTT broker}

MQTT publisher and subscriber do not run at the same time and they are not connected directly to each other. Another component called message broker comes in between and is connected to the publisher and the subscriber to receive the published messages from the publisher and distribute the messages to the subscribers accordingly [9]. MQTT can handle thousands of clients concurrently and plays as a hub in MQTT communications. In MQTT, messages are published into customizable topics, which carry meaningful information about the payload of the messages, and the subscribers need to subscribe to the defined topics. MQTT topics are hierarchical strings with different layers separated from each other by a forward slash [9]. (1) indicates a typical topic string.

\section{DataTypeName/IEDName/MicrogridName/}

MQTT is suitable for applications with resource-constrained embedded devices in the system. Even a small micro controller with a running MQTT library could be an MQTT publisher/subscriber. For local communications within a local network, HiveMQ [11] could be utilized by the IoT gateway of the MGCCs.

Multiple publishers and subscribers are implemented in this work in python scripts using Paho libraries [12]. Instead of using a local broker in this paper, an online MQTT server offered by ("iot.eclipse.org") for broader communications without geographical limitations [13] is used.

\section{Cloud Communications}

MGCCs in the proposed MMG architecture play a key role. The MGCC in each MG collects data from each IED in the MG and sends the data to the cloud. In addition, MGCCs get the results of the global energy optimization algorithm from the corresponding cloud channel and pass the results to the corresponding IED in that specific MG. In other words, the interactions with cloud from each MG is realized by MGCCs in a bi-directional way. HTTP requests such as HTTP POST and GET requests are implemented within the MGCCs over TCP/IP connections to interact with the cloud server. HTTP is an application-level protocol with default port of 80 . The interactions are realized using REST services of the cloud server.

Among all of the commercially available cloud servers, ThingSpeak [14] is chosen for the designed MMG scheme. The main advantage of using ThingSpeak in MMG architecture is the facilitated interface to MATLAB. All the data, collected in the cloud channels and received from MGCCs via MQTT, could be retrieved in MATLAB using MATLAB ThingSpeak toolbox and be post-processed. The global energy optimization algorithm runs in MATLAB interface of the cloud.

Furthermore, the time interval for data collection in ThingSpeak platform is customizable and this offers flexibilities to the platform to be compatible with both timecritical applications (e.g. protection of MGs) and non-timecritical applications (e.g. SCADA and Control of MGs). Finally, there are built-in manager blocks such as channel feed manager block and channel security manager blocks, which make ThingSpeak a reliable and secure cloud-based platform. These functional blocks are shown in the top layer of Fig. 2.

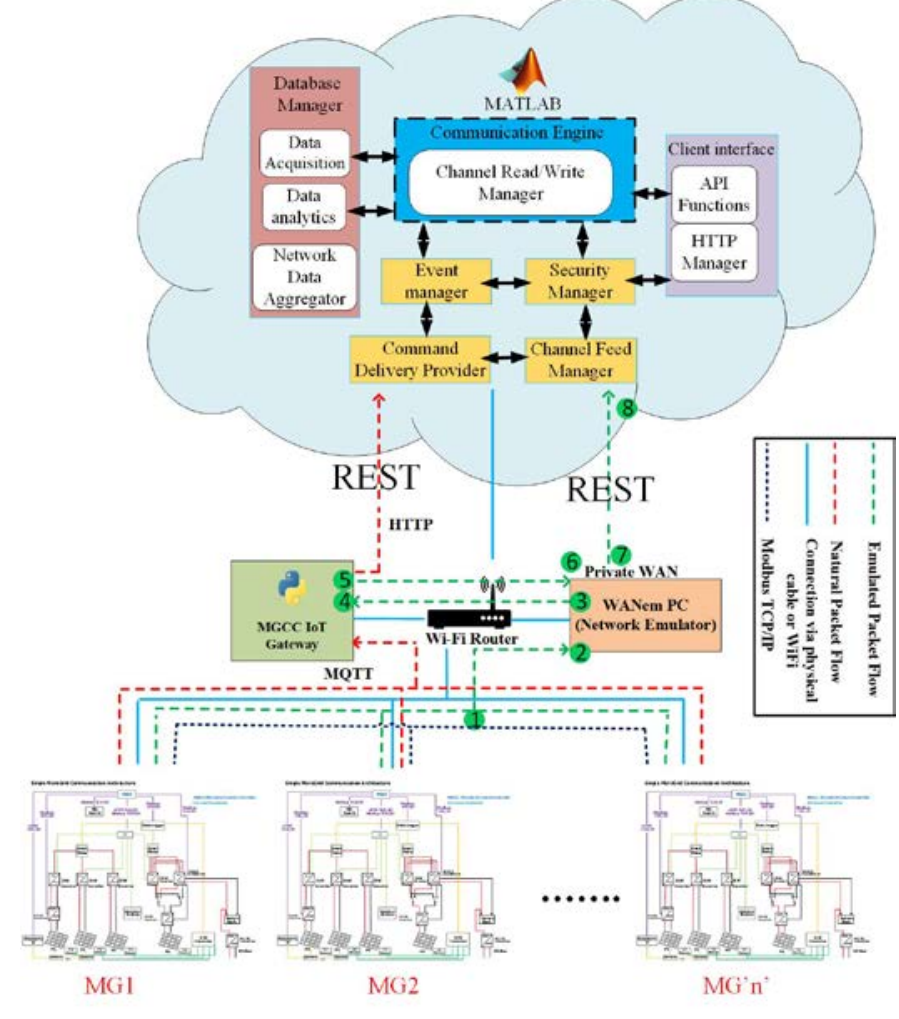

Fig. 2. Overview of the cloud-based MMG communication architecture

\section{Network Emulator Unit in the platform}

In real-world MMG case scenarios, the number of IEDs capable of communication is large. This can produce problems in centralized operation of Multiple Microgrids by causing network latency due to high number of MQTT messages in the network. Another issue in real-world scenarios is reliability of 
the communication links in different nodes of the MG. In order to address these issues, the platform utilizes a virtual Network emulator to mimic behavior of a real network. Network emulation is the act of altering the rout of the data packets in a way that a customizable latency could be added to the packet flow. This is indicated in Fig. 2.

Red dotted line in Fig. 2 represents the normal packet flow in MMG architecture without any action on network emulation. Data packets are published by IEDs in each MG and through the MGCC IoT gateway of each MG, the data is collected in the corresponding cloud channel. It must be noted that there is a separate cloud channel configured for each MG.

Green dotted line indicates the packet flow path in case of having a virtual network emulator. In this case, the data packet flow is marked in Fig. 2 by numbers from 1 to 8 . This means that data packets are not directly transferred to the cloud and they go through the WANem machine first. The WANem machine has a graphical user interface to give options to the users with customizable latencies. In the proposed architecture, there is a time delay threshold designed to switch from centralized EMS operational mode to the distributed EMS mode. In case of not receiving a data in a channel within 0.3 seconds (Round-Trip-Time (RTT) bigger than 0.3 seconds), the distributed mode, where the local controllers (MGCCs) run individual EMS algorithms and make decisions for the MG regardless of the cloud interactions, would be activated.

The functionality of the WANem machine in the proposed platform is captured by a network traffic analyzer called Wireshark software as indicated in Fig. 3. As it could be observed in Fig. 3, after filtering MQTT protocol in Wireshark, the initial round trip time (iRTT) for a published MQTT message in the platform after WANem machine is activated is 0.495286 seconds which is higher than iRTT for normal packet flow for MQTT (around 0.2 seconds). This network latency causes distributed mode to be triggered in the proposed architecture.

\section{E. EMS Cost Function}

To inspect the effect of the communication delays on the EMS performance, an objective function is defined. The objective function is designed to minimize the cost of the MMG by reducing the operation costs, the cost of the energy purchase from grid and demand charges.

$$
J_{\text {cost }}=\sum_{t=t_{0}}^{T} C_{p}(t) \cdot P_{\text {grid }}(t) \cdot \Delta t+C_{o p}(t) \cdot P_{M G}(t) \cdot \Delta t+
$$

$C_{\text {peak }} \cdot P_{\text {peak }}$

where $P_{\text {grid }}(t), P_{M G}(t)$, and $P_{\text {peak }}$ are the purchase power from grid, the power of MG, and the peak power of the month. $C_{p}(t)$, $C_{o p}(t)$, and $C_{\text {peak }}$ are the cost coefficient of the purchased energy from the grid, operating cost coefficient of the power of MG, and cost coefficient of the grid demand charges respectively. $t_{0}, T$ and $\Delta t$ represent the beginning, end and time of simulation period, respectively.

The utility charges the MGs based on Time of Use (TOU) tariffs in different categories such as retail energy usage charges and network charges. The MGs are charged for both energy usage in $\mathrm{kWh}$ on a daily basis and demand usage in kVA on a monthly basis. The charges for energy usage are applied depending on the time of the day, being peak hours or off-peak hours. The TOU tariffs for large customers in Queensland are illustrated in Table I.

TABLE I

TIME OF USE (TOU) TARIFFS FOR THE UNIVERSITY

\begin{tabular}{|c|c|c|}
\hline Charges & Unit Price & Unit \\
\hline \multicolumn{3}{|c|}{ Retail Energy Usage Charges } \\
\hline QLD Peak & 10.68 & $\mathrm{c} / \mathrm{kWh}$ \\
\hline QLD Off-peak & 5.8 & $\mathrm{c} / \mathrm{kWh}$ \\
\hline \multicolumn{3}{|l|}{ Network Charges } \\
\hline Demand Charges & 9.333 & \$/KVA/Month \\
\hline
\end{tabular}

According to Table I, the electricity costs to consider are the purchased energy cost, $C_{p}(t)$ and peak demand cost, $C_{\text {peak }}$, where $C_{p}(t)$ includes off-peak and peak energy prices and $C_{\text {peak }}$ includes demand charges. The results for the EMS algorithm in centralized and distributed operational modes are presented in the following section.

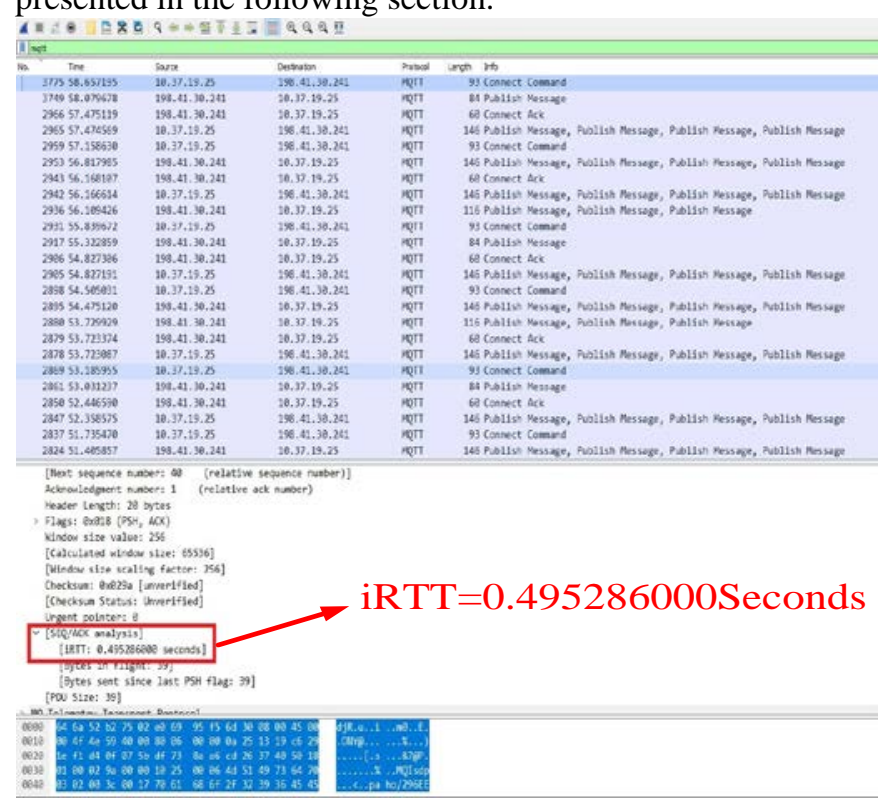

Fig. 3. MG optimization results with centralized communication failure

\section{SiMULATION RESULTS}

In this section, the impact of the communication system on the MMG is investigated. In the presence of the communication system, the MGs can share the information such as generation power and load consumption with each other. In order to reduce the operating costs of the MMG, the MGs get the required energy from the neighboring MGs instead of buying from the grid. In other words, the communication system provides the required information for the MGs to transfer energy. Using the cost optimization method in [15], the MMG total cost is achieved for the system performance comparison. Three MGs are considered for the simulation, where the MGs are modeled and simulated based on the characteristics of the MG testing facility at Griffith University [16]. The MGs are modeled identical with different load profiles. The power generation and load consumption data of three MGs are obtained from the monitoring system at GU. 


\section{A. MMG Total Cost with Centralized Communication System} Failure

The main advantage of the centralized communication system is its simplicity over the distributed communication system. However, in case of communication failure/delay, the centralized communication system of MMG would not operate properly and the MGs would not be able to communicate to each other. For this case study, the three MGs are operating under the centralized communication system and it is assumed that a communication failure/delay happened.

To minimize the MMG costs, the MGs can transfer energy to the neighbouring MGs instead of purchasing energy from the grid. In the centralized mode, the connection to the cloud is the only way for the MGs to communicate to each other. If cloud communication gets disconnected, then MGs would operate autonomously regardless of other MGs and MMG central controller. Figs. 4 to 6 illustrate the MMG optimization system operation in case of communication failure/delay. The performance of central EMS is investigated for three MGs in two different cases of optimized and not optimized operation cost. Both of the cases are simulated with the assumption of no communication to the cloud. The load profile of the MGs for the day with the highest demand in January 2018 is chosen.

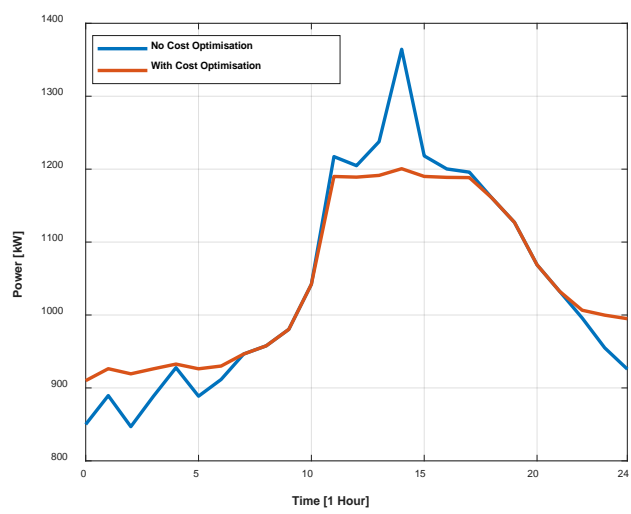

Fig. 4. MG1 optimization results with centralized communication system failure

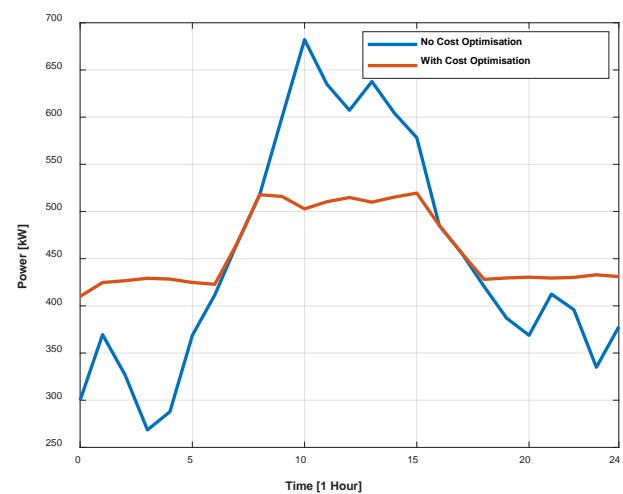

Fig. 5. MG2 optimization results with centralized communication system failure

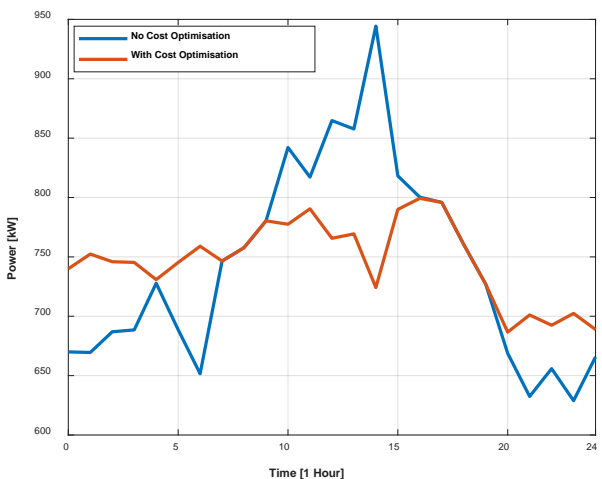

Fig. 6. MG3 optimization results with centralized communication system failure

As seen in Figs. 4 to 6, the MGs operate separately and do not transfer their surplus energy to other MGs, as there is no communication between them. Therefore, the MGs have to provide the required energy by purchasing form the grid and employing all of the resources in the MG, which would increase the operating cost of the MG. In other words, MGs cannot benefit from the energy of other MGs as the communication with the cloud is disconnected and consequently each MG optimizes independently.

\section{B. MMG Total Cost with Distributed Communication System}

The proposed distributed communication system for the MMG is capable of transferring data between the MGs even in case of cloud communication failure. In other words, the MGs get the required information from the cloud as well as directly from the neighboring MGs. In the communication architecture, if cloud connection is failed (e.g. delay longer than 0.3 seconds), then the system transfers to the distributed mode where MGs are connected via Modbus TCP/IP to each other. Therefore, the MGs can transfer the surplus energy to the neighboring MGs and reduce the operating costs of the MMG. Figs. 7 to 9 indicate the performance of the optimization system to minimize the costs employing the proposed distributed communication system.

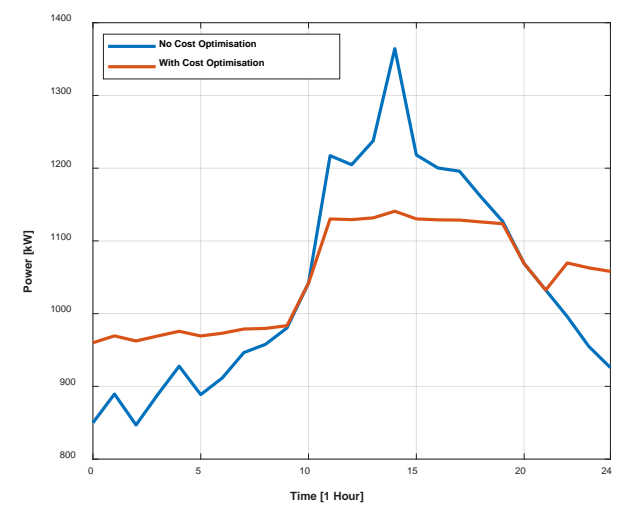

Fig. 7. MG1 optimization results with distributed communication 


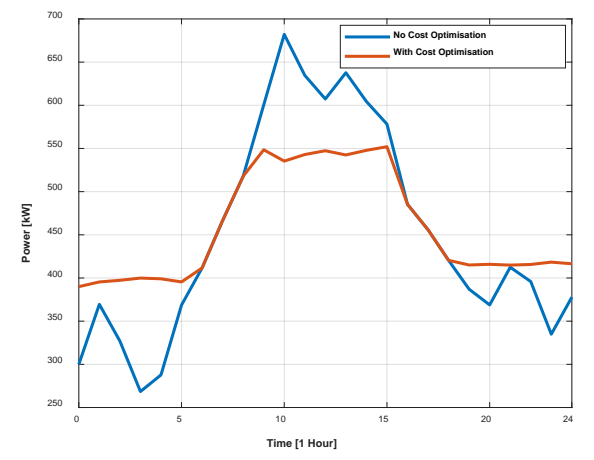

Fig. 8. MG2 optimization results with distributed communication

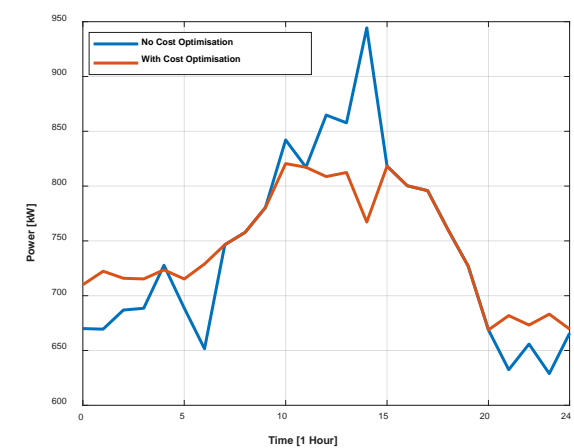

Fig. 9. MG3 optimization results with distributed communication

With the distributed communication system, it can be seen that the optimization system of the MMG gets the surplus energy of MG2 and MG3 to reduce the operating costs of MG1. Furthermore, the share of MG2 and MG3 is decreased in order to minimize the total cost of the MMG. Table II compares the total cost of the MMG for case 1) no distributed communication system and case 2) activation of the distributed communication. TABLE II

OPERATING COST OF MMG IN CASE OF FAILURE OF THE CENTRALIZED

\begin{tabular}{ccc}
\hline \multicolumn{3}{c}{ COMMUNICATION SYSTEMS } \\
$\begin{array}{c}\text { Case 1) Centralized } \\
\text { Communication } \\
\text { System failure }\end{array}$ & $\begin{array}{c}\text { Case 2) Distributed } \\
\text { Communication } \\
\text { System }\end{array}$ \\
\hline \hline MG1 Total Cost & $\$ 734 \mathrm{k}$ & $\$ 702 \mathrm{k}$ \\
\hline MG2 Total Cost & $\$ 356 \mathrm{k}$ & $\$ 361 \mathrm{k}$ \\
\hline MG3 Total Cost & $\$ 500 \mathrm{k}$ & $\$ 506 \mathrm{k}$ \\
\hline MMG Total Cost & $\$ 1590 \mathrm{k}$ & $\$ 1569 \mathrm{k}$ \\
\hline
\end{tabular}

From Table II, it is seen that the total cost of MMG in case of the communication system failure/delay in the cloud connection is $\$ 21 \mathrm{k}$ less if the distributed system is activated compared to the centralized communication system with no distributed communication system and MGs operating independently.

\section{CONCLUSION}

This paper presents a novel hierarchical communication platform with a two-level structure, which is suitable for MultiMMG management. The proposed platform utilizes Modbus TCP/IP for local MG data exchange and as a backup communication method among MGs in case of a failure in the cloud level communication. MQTT publisher/subscriber is adopted for cloud level messaging and HTTP TCP/IP for interactions between the platform and a cloud-server. The cost analysis provided in the simulation results section shows efficiency of the proposed distributed communication platform in comparison with the centralized operation of the MMG communications. It can be mentioned that the optimized cost of the MMG in case of centralized communication failure is less when the distributed communication system is activated than the situation that each MG operates independently.

\section{REFERENCES}

M. Moghimi, C. Bennett, D. Leskarac, S. Stegen, and L. Junwei, "Communication architecture and data acquisition for experimental MicroGrid installations," in Power and Energy Engineering Conference (APPEEC), 2015 IEEE PES Asia-Pacific, 2015, pp. 15.

S. Mittal, M. Ruth, A. Pratt, M. Lunacek, D. Krishnamurthy, and W. Jones, "A system-of-systems approach for integrated energy systems modeling and simulation," presented at the Proceedings of the Conference on Summer Computer Simulation, Chicago, Illinois, 2015.

P. Jamborsalamati, E. Fernandez, M. J. Hossain, and F. H. M. Rafi, "Design and implementation of a cloud-based IoT platform for data acquisition and device supply management in smart buildings," in 2017 Australasian Universities Power Engineering Conference (AUPEC), 2017, pp. 1-6.

N. Hatziargyriou and Ieee, Microgrid: architectures and control (no. Book, Whole). Chichester, West Sussex, United Kingdom: John Wiley \& Sons Inc, 2013.

P. Jamborsalamati, A. Sadu, F. Ponci, and A. Monti, "A flexible HiL testing platform for performance evaluation of IEC 61850-based protection schemes," in 2016 IEEE Power and Energy Society General Meeting (PESGM), 2016, pp. 1-5.

P. Jamborsalamati, A. Sadu, F. Ponci, and A. Monti, using IEC 61850 in active distribution grids," in 2015 International Conference on Renewable Energy Research and Applications (ICRERA), 2015, pp. 606-611.

F. B. Costa, A. Monti, F. V. Lopes, K. M. Silva, P. Jamborsalamati, and A. Sadu, "Two-Terminal Traveling-Wave-Based TransmissionLine Protection," IEEE Transactions on Power Delivery, vol. 32, no. 3, pp. 1382-1393, 2017.

A. Bani-Ahmed, L. Weber, A. Nasiri, and H. Hosseini, "Microgrid IEEE.

Available: http://mqtt.org/

S. S. Thale, R. G. Wandhare, and V. Agarwal, "A Novel Reconfigurable Microgrid Architecture With Renewable Energy Sources and Storage," Industry Applications, IEEE Transactions on, vol. 51, no. 2, pp. 1805-1816, 2015.

Available: http://www.hivemq.com/

Available: http://www.eclipse.org/paho/

Available: http://thingspeak.com/

M. Moghimi, R. Garmabdari, S. Stegen, J. Lu, "Battery Energy Storage Cost and Capacity Optimization for University Research Center," presented at the 2018 IEEE Industrial \& Commercial Power Systems (I\&CPS) Technical Conference, Niagara Falls, ON, Canada, 2018.

D. Leskarac, C. Bennett, M. Moghimi, S. Stegen, and L. Junwei, "Testing facility for research and development of Smart-MicroGrid technologies," in Power and Energy Engineering Conference (APPEEC), 2015 IEEE PES Asia-Pacific, 2015, pp. 1-5. "Implementation of an agent based distributed FLISR algorithm communications: State of the art and future trends," pp. 780-785:

Available: https://iot.eclipse.org/ 